\title{
Pathogenicity of autoantibodies in neurological disorders
}

\section{Thashi Chang}

Journal of the Ceylon College of Physicians, 2012, 43, 7-14

Index words: autoantibodies, GAD antibodies, stiffperson syndrome (SPS), GABAergic neurons, CNS

\section{Introduction}

Over the last two decades an increasing number of neurological disorders have been found to be associated with autoantibodies (Table 1). A pathogenic role for autoantibodies has been postulated in many of these neurological disorders, but established only in some of the diseases of the peripheral nervous system $^{1}$. The increasing recognition of pathogenic autoantibodies in neurological disorders has transformed previously incurable conditions to disorders that respond to active immunological intervention with potential for cure.

Table 1. Autoantibody-associated neurological disorders

Neurological disorder Target of autoantibody

\section{Peripheral nervous system (PNS)}

Myasthenia gravis

Lambert-Eaton myasthenic syndrome

Guillain-Barre syndrome

Multifocal motor neuropathy

Acquired neuromyotonia

Monoclonal gammopathy associated neuropathy

Central nervous system (CNS)

Stiff person syndrome

Neuromyelitis optica (Devic disease)

Multiple sclerosis

Limbic encephalitis

NMDAR-Ab encephalitis

Rasmussen encephalitis

Bieckerstaff's encephalitis

PNS and CNS

Paraneoplastic syndromes
NAChR, MuSK

VGCC P/Q-type

GM1, GD1b, GQ1b

GM1

VGKC

MAG

GAD

Aquaporin 4

MBP, MOG, PLP, OSP

Hu, Ma2, VGKC, CASPR2, LGI1, AMPAR, GABABR, GAD

NMDAR

GluR3

GQ1b

$\mathrm{Hu}$, Yo, Tr, Ma2, Ri, Amphiphysin

\footnotetext{
${ }^{1}$ Department of Clinical Medicine, University of Colombo, Sri Lanka.
} 
The mere presence of circulating autoantibodies is inadequate to define an autoimmune aetiology, since such antibodies can be generated as an epiphenomenon secondary to tissue destruction. A pathogenic role for autoantibodies can be inferred from clinical improvement with plasmapheresis, but certain, intravenous immunoglobulins, monoclonal antibodies and immunosuppressant therapy will be effective only in disorders in which the autoantibodies are truly pathogenic.

Recently, a number of central nervous system (CNS) disorders, such as stiff person syndrome, limbic encephalitis, NMDARAb encephalitis, some forms of epilepsy, neuromyelitis optica and cerebellar ataxia have been found to associate with specific serum autoantibodies. Immunotherapy has proven effective in ameliorating these disorders, previously considered not to have effective treatment beyond symptomatic relief. However, the exact role of autoantibodies in these diseases remains unclear although circumstantial evidence suggests that they may be pathogenic. Understanding the underlying disease mechanisms would enable the development of specific immune interventions that may be more effective and have less adverse effects related to non-specific immune modulation.

\section{Pathogenicity of autoantibodies in autoimmune diseases}

Stringent criteria need to be met to prove a pathogenic role of serum autoantibodies. These criteria have been drawn up modelled on Koch's postulates ${ }^{2}$ and recently revised ${ }^{3}$, and are summarised in Table 2. It is believed that the study of the antibody mediated mechanisms underlying the stiff person syndrome would serve as a paradigm in understanding the disease mechanisms in the recently emerged antibodyassociated CNS diseases.

\section{Table 2. Modified Koch's postulates for antibody-mediated disease}

Autoantibodies to functionally important protein

Plasmapheresis produces marked clinical improvement

Transfer of disease by placental transfer of antibodies to the baby

Transfer of disease by injection of patient serum immunoglobulins into mice

Demonstration of functional effects of antibodies using appropriate cell lines in vitro

\section{Stiff person syndrome}

Stiff person syndrome (SPS) is a highly disabling, progressive disorder of the central nervous system characterised by muscular rigidity and spasms. SPS arises in the fourth and fifth decades of life and is twice as commoner in women. The onset is insidious. Usually symmetrical, continuous stiffness characterised by tight, stony-hard, board-like muscles occur, most prominent in axial (abdominal and thoracolumbar paraspinals) and proximal limb muscles. Increased tone of the paraspinal muscles gives rise to hyperlordosis, a hallmark diagnostic sign in SPS. Superimposed upon this persistent rigidity of muscles, sudden stimuli often precipitate paroxysms of violent muscle spasms.

Approximately $10 \%$ have associated epilepsy 4 . Approximately 30 to $40 \%$ of SPS patients develop type 1 diabetes mellitus (T1DM) ${ }^{5,6}$. An additional $40 \%$ have at least one of a number of organ-specific autoimmune disorders or organ-specific autoantibodies ${ }^{5}$. In a few cases, thymoma has been associated with $\operatorname{SPS}^{7,8}$.

The presence of autoantibodies to glutamic acid decarboxylase (GADAbs) in stiff person syndrome was discovered 20 years ago ${ }^{9}$. Since then GADAbs have been reported in approximately 60 to $80 \%$ of patients with SPS. A high titre is thought to distinguish GADAbs in SPS from GADAbs infrequently reported in other neurological diseases-thymoma $(5 \%)$ and normal healthy controls $(1 \%)^{10}$. Although high titres of GADAb in the range seen in SPS have been reported in some cases of autoimmune polyglandular syndrome, idiopathic late-onset cerebellar ataxia and drugresistant localisation-related epilepsy ${ }^{11,12}$, the prevalence of GADAbs in these diseases are rare. However, GADAbs are detected in up to $80 \%$ of patients with T1DM where it is both a marker of disease and predictor of risk ${ }^{13}$, but the titre is considered to be much lower than that of SPS ${ }^{11}$, although differences in reactivity with GAD epitopes has also been suggested.

SPS remains a clinical diagnosis supported by typical EMG findings and positive serology for GADAbs.

Symptomatic relief is often achieved with agents that enhance GABAergic transmission. Diazepam, and Baclofen, $\mathrm{GABA}_{B}$ agonists are first-line in treatment. However, the doses required are intolerably high thus limiting their benefit.

In a randomised, double-blinded, placebocontrolled crossover trial, Dalakas and coworkers demonstrated significant improvement in objective stiffness parameters and activities of daily living in GADAb-positive classical SPS patients treated with 
intravenous immunoglobulin (IVIg) ${ }^{14}$. Plasmapheresis has been beneficial in 6 of 11 reported cases ${ }^{15}$. Corticosteroids have been successful in some patients with $\mathrm{SPS}^{4}$, but no controlled trial has been conducted to date. Benefit in individual cases with immunosuppressive agents such as rituximab, azathioprine, methotrexate, cyclophosphomide and mycophenolate mofetil have been reported but these drugs have not been validated systematically ${ }^{16,17}$.

\section{The autoimmune hypothesis and GADAbs in SPS}

An autoimmune pathogenesis of SPS has been suggested because of: the association of SPS with autoantibodies, predominantly to GAD; the association of SPS with other autoimmune diseases; a female preponderance; and the clinical response of SPS to immunotherapy. However, whether GADAbs are primarily responsible for the pathogenesis of disease or simply represent an event downstream from the primary cause remains unknown.

Circumstantial evidence supports a pathogenic role for GADAbs: GAD is the rate-limiting enzyme in the synthesis of the inhibitory neurotransmitter GABA, and therefore the impairment of GAD is consistent with rigidity and spasms as a consequence of impaired motor inhibition; GAD activity and GABA synthesis as well as presynaptic GABA transmission, are inhibited in vitro by GADAb-positive IgG from patients with SPS; GADAbs are produced intrathecally; mean GABA levels in the CSF are lower in patients with SPS; enhanced excitability and low GABA levels in the motor cortex are evident in patients with SPS; some SPS patients respond to plasmapheresis.

However, an argument against a pathogenic role for GADAbs has long been that these autoantibodies recognise an intracellular antigen. The ability to recognise antigenic targets expressed on the cell surface is a pre-requisite for pathogenicity of antibodies. It has been suggested that certain peptide fragments of GAD may be expressed on the cell surface during exocytosis of $\mathrm{GABA}^{18}$, but to date there have not been any experimental evidence to substantiate this. Furthermore, evidence against a direct pathogenic role for GADAbs has included a report of two offspring who acquired high titres of GADAbs transplacentally from their mother, who had SPS, but did not develop signs of the disease ${ }^{19}$. However, the failure of this 'natural passive transfer' needs to be considered in the context of an intact blood brain barrier in the offspring that would have prevented access of antibodies into the CNS, and also that in diseases believed to be caused by transplacental antibody transfer, such as congenital heart block, the risk in the fetus was only $2 \%$ in mothers who were seropositive for antibodies ${ }^{20}$.

With the objective of defining the role of GADAbs in SPS, this study was designed to characterise GAD antibodies in SPS and test whether GADAbs possessed the characteristics necessary for pathogenicity or whether SPS had other autoantibodies that were pathogenic whilst GADAbs merely represented a secondary epiphenomenon.

\section{Patients}

Sera from 25 patients clinically diagnosed with SPS or its variants were selected. The patients were mainly female, with ages ranging from 32 to 80 years at time of study, and a mean duration of disease of 40 months. Three patients had associated epilepsy, one had cerebellar ataxia and one had had optic neuritis. Ten patients $(40 \%)$ had associated diabetes mellitus, 6 patients had other autoimmune diseases and 4 patients had serum autoantibodies without evident autoimmune disease.

As a comparison group, sera from 4 patients with epilepsy and sera from 5 patients with T1DM were selected on the basis of being positive for GADAbs in previous studies.

\section{GAD antibodies}

The radioimmunoprecipitation assay for GAD antibodies was positive in the sera of 19 of the total SPS cohort (76\%). The GADAb titre in SPS was $>1000$ $\mathrm{U} / \mathrm{ml}$ in most patients (only 4 of the 19 seropositive SPS had titres < $1000 \mathrm{U} / \mathrm{ml}$ ) (Figure 1). In contrast, the titres of the diabetic sera were much lower than those of SPS, but the epilepsy sera that were selected had high titers in the same range as SPS. On immunohistochemistry (where patient sera was reacted on rat brain sections and stained to detect human antibodies binding to brain antigens), 13 of the 25 SPS sera (52\%) bound to rat brain tissue in a manner that very largely co-localised with binding of monoclonal antibodies to $\mathrm{GAD}^{21}$. All of the diabetic sera were negative.

Pathogenic antibodies, like those to the acetylcholine receptor $(\mathrm{AChR})$ in myasthenia gravis appear to be high affinity and IgG1 subclass. The affinity of the GAD antibodies was determined in each serum by binding of a limiting amount of serum to increasing concentrations of ${ }^{125} \mathrm{I}-\mathrm{GAD}$ and deriving the dissociation constant $(\mathrm{Kd})$ from plotting saturation binding curves and performing Scatchard analysis 
(Figure 2). A lower $\mathrm{Kd}$ denotes a higher affinity. All GAD antibodies showed high affinity binding with $\mathrm{Kd}$ values ranging from $0.06-0.78 \mathrm{nM}$. The majority of patients, irrespective of their clinical condition, had IgG1 antibodies, which are capable of activating complement as a mechanism of neuronal cell damage (Figure 3). These results suggest that GADAbs in SPS possess characteristics of pathogenic antibodies such as high specificity for disease at high titre, high affinity to its antigen (GAD) and being predominantly lgG1 that is capable of activating complement.

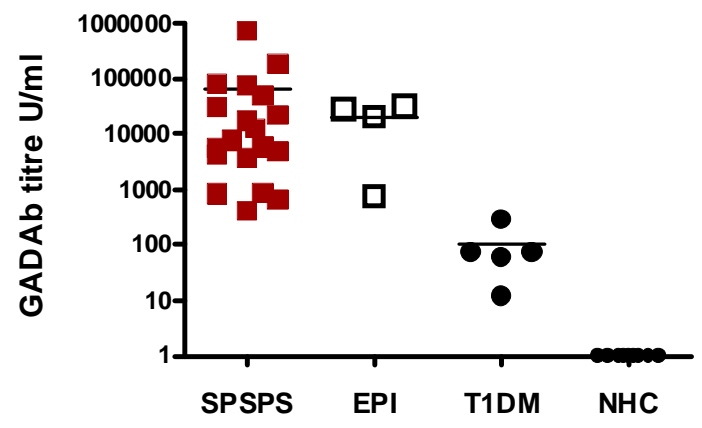

Figure 1. GADAb titres in patients with SPSPS $(n=19)$, epilepsy $(n=4), \operatorname{T1DM}(n=5)$ and normal healthy controls $(n=9)$. Note the log scale for GADAb titres. The normal range for GADAbs in healthy individuals is $<1 \mathrm{U} / \mathrm{ml}$. SPSPS=seropositive SPS; EPI=epileptic patients; $\mathrm{NHC}=$ normal healthy controls.

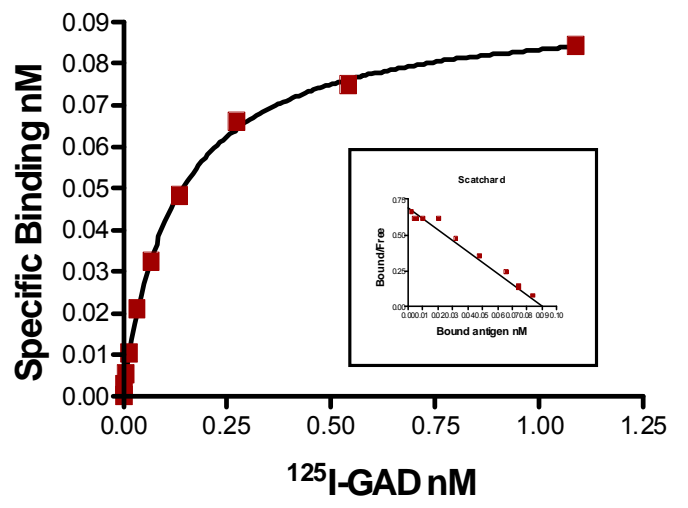

Figure 2. Representative saturation binding curve and Scatchard plot (inset) for a SPSPS patient serum.

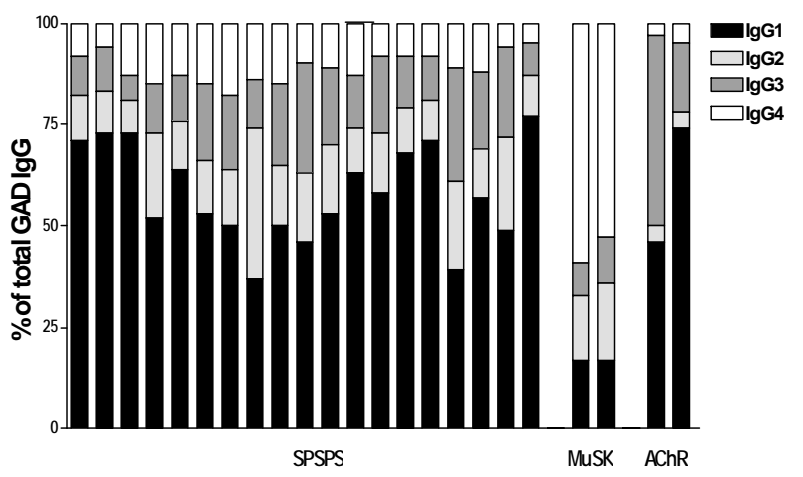

Figure 3. GADAb IgG subclasses in sera of patients with SPSPS ( $n=19)$. As controls, IgG subclasses of MuSK antibodies and IgG subclasses of AChR antibodies were tested in sera from 4 patients with MG.

\section{Binding of autoantibodies to neuronal cells}

To test whether GADAbs bind to epitopes expressed on the membrane of neuronal cells, SPS patient sera were reacted with three neuroblastoma cell lines and looked for binding by flow-cytometry (Figure 4). There was generally higher binding of the SPS sera than of the control sera, but only few sera bound strongly.
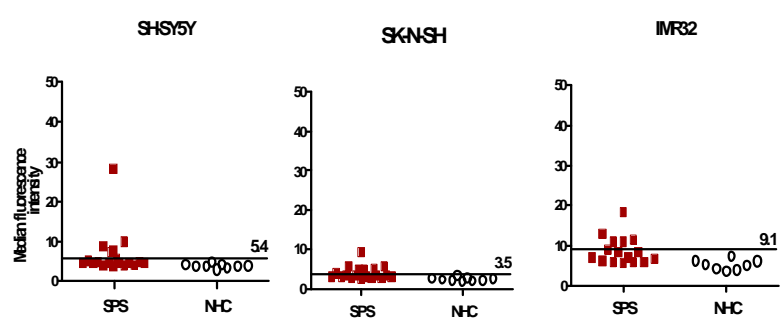

Figure 4. Flow cytometric measurement of antibody binding to cultured neuroblastoma cells (SH-SY5Y, SK$\mathrm{N}-\mathrm{SH}$ and IMR-32) with sera from stiff-person syndrome (SPS) patients and normal healthy controls (NHC). 9 of the 19 SPS sera tested showed binding to one or more neuroblastoma cell lines. The horizontal line in each graph indicates the cut-off for positive results defined as the mean MFI of NHC + 3SD.

\section{Passive transfer}

Passive transfer of the disease into experimental animals forms a critical step in establishing a pathogenic role for autoantibodies. Two matchedgroups of mice were injected with either plasma IgG from patients with SPS or with IgG from healthy controls. Human GADAb titres in mice passively 
transferred with SPS IgG reached levels comparable to that of human SPS. However, immunisation did not produce a disease phenotype reminiscent of human SPS in mice. Immunohistochemical, immunofluorescence and stereology analysis did not show any evidence of inflammation or GABAergic neuronal loss in mice injected with human SPS IgG.

The passive transfer of SPS IgG into mice failed to model the disease seen in humans either phenotypically or pathologically. Whether inducing a cellular response, in addition to a humoral response, and whether a longer duration of exposure to GADAbs are important factors in reproducing SPS in mice was addressed by actively immunising mice with GAD antigen.

\section{Active immunisation}

The test group of GAD65-EGFP transgenic mice were immunised with full-construct recombinant human (rh) GAD65. A sustained immune response was maintained over 5 months with repeated boosters of rhGAD65. The control group was immunised with the inert phosphate buffered saline (PBS). A clinical phenotype was sought by periodical behavioural analysis, whilst pathological correlates were sought by immunohistological analysis of the brain and spinal cord for an inflammatory cellular infiltrate or GABAergic neuronal loss.

Ten days after the second booster, the GADAb titre in mice immunised with rhGAD65 ranged from 350 to $32428 \mathrm{U} / \mathrm{ml}$ (mean 16386) with only one serum $<1000 \mathrm{U} / \mathrm{ml}$. A high antibody titre was sustained over a period of 5 months although there was a drop with time from the last booster immunisation (Figure 5). None of the mice immunised with PBS had GADAbs at any of the three time points. Despite the high titre GADAb response, none of the mice had glucose in their urine on analysis with reagent strips.

To further characterise serum antibodies from mice immunised with rhGAD65, the mouse sera were immunoreacted with primary neuronal cultures derived from GAD65-EGFP transgenic mice. When cultures were permeabilised mouse serum antibodies were seen binding intracellularly to all EGFP-expressing neurons (Figure 6a). Pre-incubation with rhGAD65 completely adsorbed out the intracellular binding antibodies, but surface-binding antibodies were evident in a few EGFPand non-EGFP-expressing neurons (Figure 6b). When immunoreacted with unpermeabilised cultures, mouse serum antibodies were seen binding to the surface of neurons (Figure 6c). These neuronal surface binding antibodies in mouse serum were not adsorbed by preincubating with rhGAD65 (Figure 6d). In contrast to GAD-immunised mice sera, PBS-immunised mice sera did not show any immunoreactivity to either permeabilised or unpermeabilised neurons in primary cerebellar cultures. These results suggest that immunisation with GAD induced GADAbs that bound intracellularly and other (non-GAD) antibodies that bound to the surface of neurons. This is known as determinant spreading where one antigen provokes an immune response to other antigens with similar epitopes.

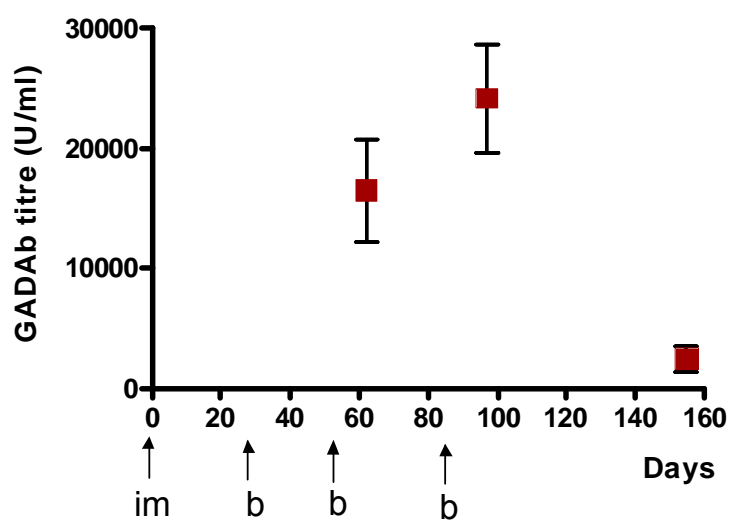

Figure 5. Mean GADAb titres in mice immunised with rhGAD65, 10 days after the second booster (D62, n=9), 15 days $(D 97, n=4)$ and 73 days (D155, n=5) after the third booster. Three booster immunisations (b) were given at 4-week intervals after the initial immunisation (im). All mice immunised with PBS in the control arm had a GADAb titre of $<1 \mathrm{U} / \mathrm{ml}$ at all three time points.

Active immunisation did not produce a disease phenotype in mice. However, on immunohistological analysis of brains and spinal cords one of the mice from the test group showed an inflammatory cell infiltrate (CD3 and CD45-positive cells) in the subcortical and brainstem regions Brains and spinal cord sections from mice from the test group and the control group were analysed for neuronal loss. GABAergic neurons in the brainstem was notably lower amongst GAD immunised mice, $(p=0.036)$, whilst there was no difference in total neurons in the two groups (Figure 7).

This finding was biologically significant because a loss of GABAergic inhibitory neurons in the brainstem could explain an increased excitability of spinal motor neurons that would result in axial rigidity and muscle spasms seen in SPS ${ }^{22-24}$. The absence of clinical manifestations in the presence of GABAergic neuronal loss in the brainstem of mice may be because the magnitude of the GABAergic neuronal loss did not reach the threshold required in mice to manifest axial rigidity and spasms. 
a

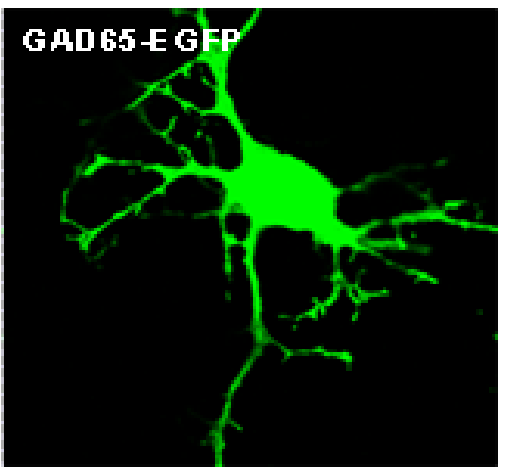

b

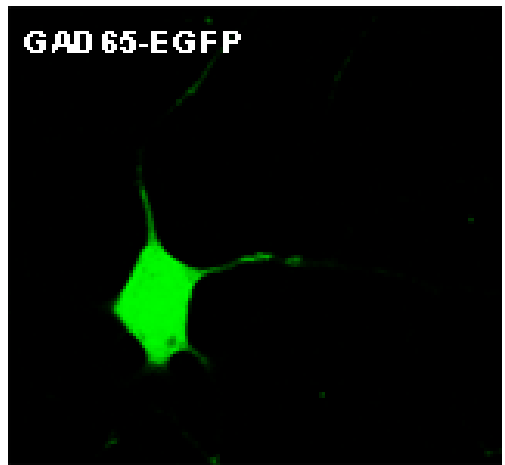

c

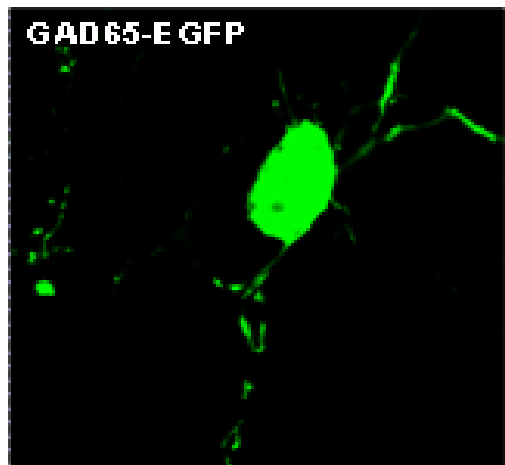

d

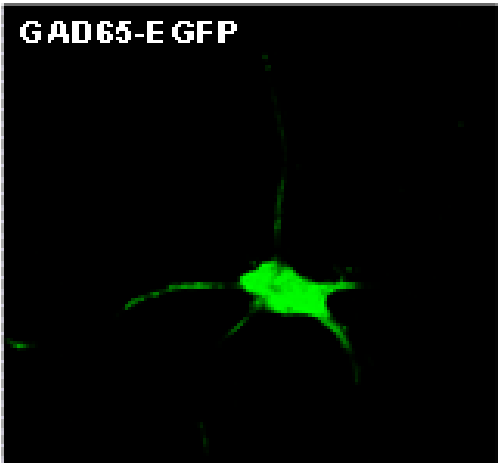

\section{GAD Mo serum}
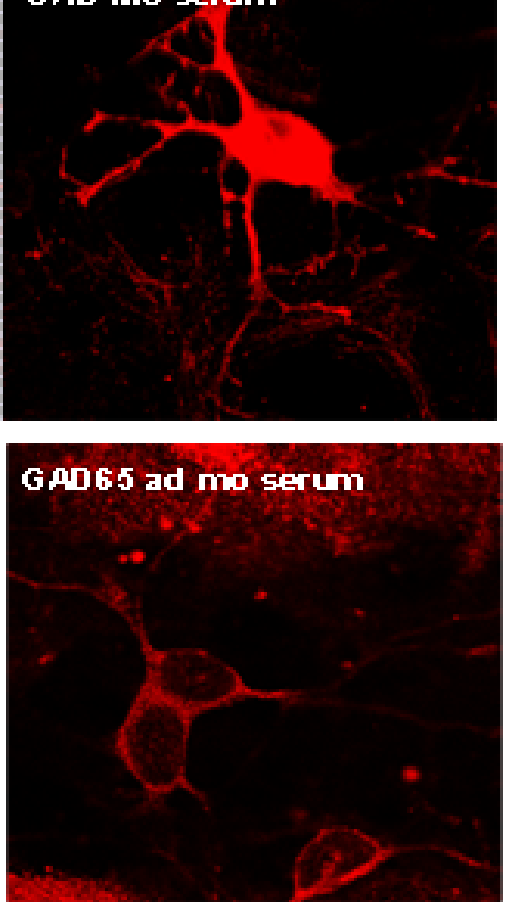

\section{GAD Mlo serum}
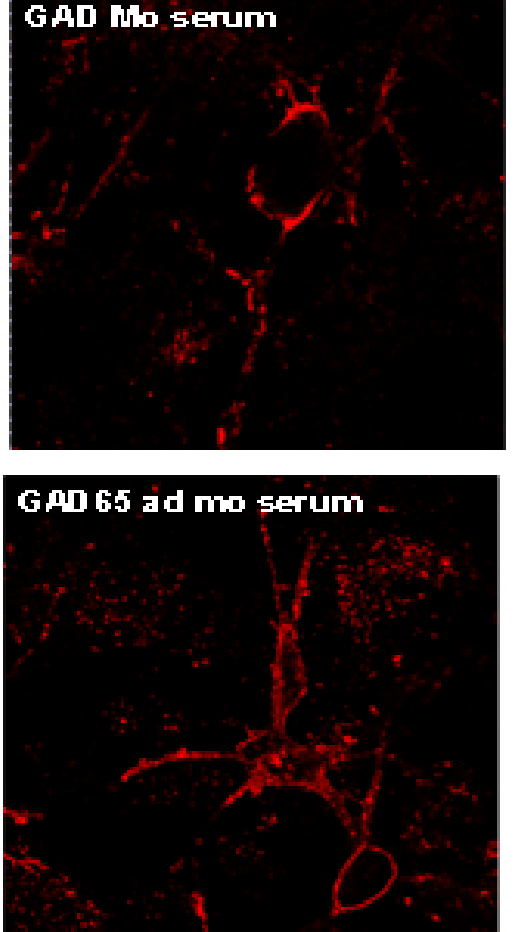
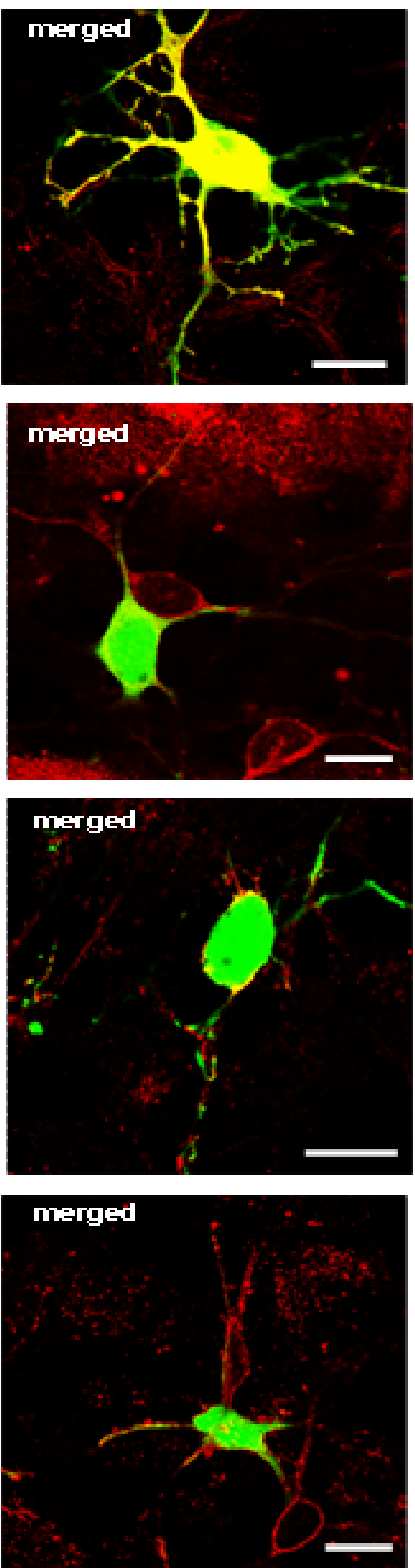

Figure 6. Confocal images of GADAb-positive mouse serum binding to cerebellar neurons in primary culture derived from GAD65-EGFP transgenic mice. (a) mouse serum antibodies bind intracellularly to permeabilised neurons. (b) After adsorption against GAD65, the same serum binds the surface of permeabilised neurons. (c) Mouse serum antibodies bind to the surface of unpermeabilised neurons. (d) Serum antibodies continue to demonstrate surface binding to unpermeabilised neurons after adsorption against GAD65. Scale bar $=10 \mu \mathrm{m}$. 

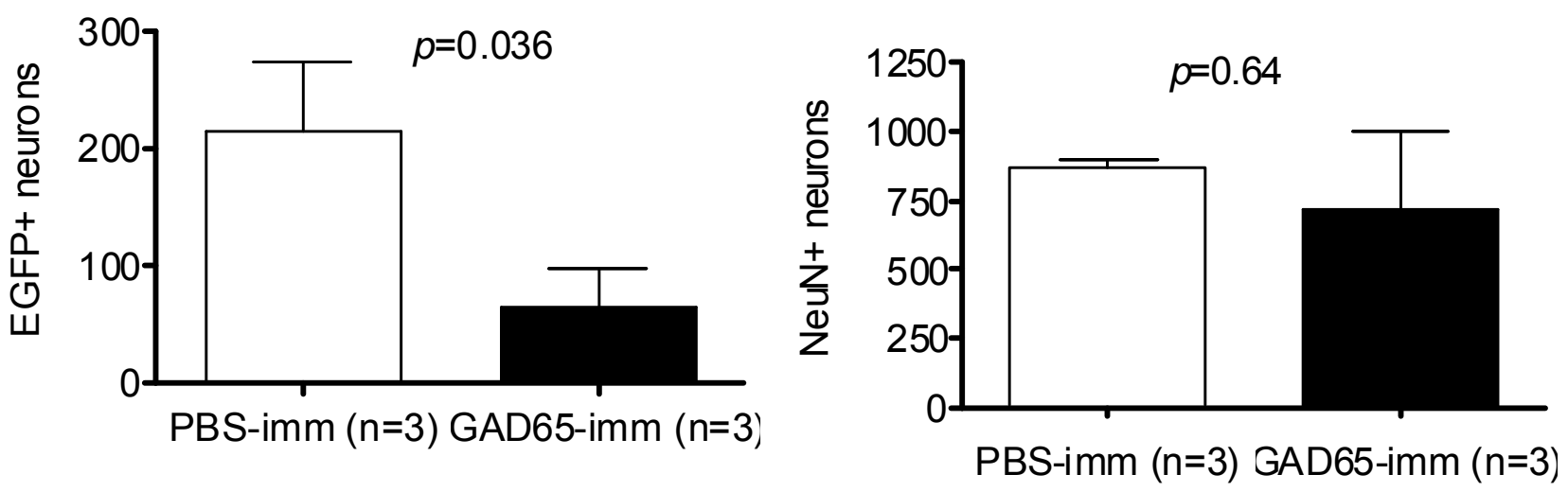

Figure 7. Column bars with means and SEMs are shown for EGFP-expressing neurons (left) and NeuN-positive neurons (right) in the brainstem of mice immunised with rhGAD65 (tests) or PBS (controls). GABAergic neurons were lower in the test mice $(p=0.036)$.

\section{Conclusions}

Using SPS as a paradigm of antibody-associated CNS disease, this study demonstrates that GADAbs in SPS are high affinity, predominantly lgG1 subclass and bind to GABAergic neurons throughout the central nervous system. There was evidence that SPS sera contain other autoantibodies that are capable of binding to cell surface determinants, but a short trial of passive transfer of GADAb-positive IgG from SPS patients did not succeed in inducing any signs of disease in recipient mice. However, interestingly, similar cellsurface antibodies were found in sera of mice immunised against GAD suggesting that they may arise secondary to GAD immunity. Although GADimmunised mice did not show clinical signs, there was loss of GABAergic neurons in the brainstem. It is possible, therefore, that a cellular immune response against GAD leads not only to high GADAbs but also to an additional humoral response towards GABAergic neurons and that both cellular and humoral responses are required to produce the cell loss that underlies the clinical features of SPS.

\section{References}

1. Vincent A. Unravelling the pathogenesis of myasthenia gravis. Nature Reviews Immunology 2002; 2: 797-804.

2. Witebsky E, Rose NR, Terplan K, Paine JR, Egan RW. Chronic thyroiditis and autoimmunization. Journal of the American Medical Association 1957; 164: 1439-47.

3. Rose NR, Bona C. Defining criteria for autoimmune diseases (Witebsky's postulates revisited). Immunology Today 1993; 14: 426-30.

4. Meinck HM, Thompson PD. Stiff man syndrome and related conditions. Movement Disorders 2002; 17: 853-66.
5. Dalakas MC, Fujii M, Li M, McElroy B. The clinical spectrum of anti-GAD antibody-positive patients with stiff-person syndrome. Neurology 2000; 55: 1531-5.

6. Solimena M, Folli F, Aparisi R, Pozza G, De Camilli P. Autoantibodies to GABA-ergic neurons and pancreatic beta cells in stiff-man syndrome. New England Journal of Medicine 1990; 322: 1555-60.

7. Nicholas AP, Chatterjee A, Arnold MM, Claussen GC, Zorn GL, Jr., Oh SJ. Stiff-persons' syndrome associated with thymoma and subsequent myasthenia gravis. Muscle Nerve 1997; 20: 493-8.

8. Tanaka H, Matsumura A, Okumura M, Kitaguchi M, Yamamoto S, luchi K. Stiff man syndrome with thymoma. Annals of Thoracic Surgery 2005; 80: 739-41.

9. Solimena M, Folli F, Denis-Donini S, Comi GC, Pozza G, De Camilli $P$, et al. Autoantibodies to glutamic acid decarboxylase in a patient with stiff-man syndrome, epilepsy, and type I diabetes mellitus. New England Journal of Medicine 1988; 318: 1012-20.

10. Meinck HM, Faber L, Morgenthaler N, Seissler J, Maile S, Butler $\mathrm{M}$, et al. Antibodies against glutamic acid decarboxylase: prevalence in neurological diseases. Journal of Neurology Neurosurgery and Psychiatry 2001; 71: 100-3.

11. Honnorat J, Saiz A, Giometto B, Vincent A, Brieva L, de Andres $C$, et al. Cerebellar ataxia with anti-glutamic acid decarboxylase antibodies - Study of 14 patients. Archives of Neurology 2001; 58: 225-30.

12. Peltola J, Kulmala P, Isojarvi J, SaizA, Latvala K, Palmio J, et al. Autoantibodies to glutamic acid decarboxylase in patients with therapy-resistant epilepsy. Neurology 2000; 55: 46-50.

13. Tuomilehto J, Zimmet P, Mackay IR, Koskela P, Vidgren G, Toivanen L, et al. Antibodies to glutamic acid decarboxylase as predictors of insulin-dependent diabetes mellitus before clinical onset of disease. Lancet 1994; 343: 1383-5. 
14. Dalakas MC, Fujii M, Li M, Lutfi B, Kyhos J, McElroy B. High-dose intravenous immune globulin for stiff-person syndrome. New England Journal of Medicine 2001; 345: 1870-6.

15. Vasconcelos OM, Dalakas MC. Stiff-person Syndrome. Current Treatment Options in Neurology 2003; 5: 79-90.

16. Baker MR, Das M, Isaacs J, Fawcett PR, Bates D. Treatment of stiff person syndrome with rituximab. Journal of Neurology Neurosurgery and Psychiatry 2005; 76: 999-1001.

17. Hao W, Davis C, Hirsch IB, Eng LJ, Daniels T, Walsh D, et al. Plasmapheresis and immunosuppression in stiff-man syndrome with type 1 diabetes: a 2-year study. Journal of Neurology 1999; 246: 731-5.

18. Levy LM, Dalakas MC, Floeter MK. The stiff-person syndrome: an autoimmune disorder affecting neurotransmission of gamma-aminobutyric acid. Annals of Internal Medicine 1999; 131: 522-30.

19. Nemni R, Caniatti LM, Gironi M, Bazzigaluppi E, De Grandis D. Stiff person syndrome does not always occur with maternal passive transfer of GAD65 antibodies. Neurology 2004; 62: 2101-2.

20. Buyon JP, Clancy RM. Maternal autoantibodies and congenital heart block: mediators, markers, and therapeutic approach. Seminars in Arthritis and Rheumatism 2003; 33: 140-54.

21. Chang T, Lang B. GAD antibodies in stiff-person syndrome. Neurology 2004; 63: 1999-2000.

22. Holstege JC. Ultrastructural evidence for GABAergic brain stem projections to spinal motoneurons in the rat. Journal of Neuroscience 1991; 11: 159-67.

23. Reichling DB, Basbaum Al. Contribution of brainstem GABAergic circuitry to descending antinociceptive controls: I. GABA-immunoreactive projection neurons in the periaqueductal gray and nucleus raphe magnus. Journal of Comparative Neurology 1990; 302: 370-7.

24. Schwartz-Giblin S, McCarthy MM, Robbins A. The medullary reticular formation is a site of muscle relaxant action of diazepam on deep back and neck muscles in the female rat. Brain Research 1996; 710: 178-88. 